time; 16 centred on $-85^{\circ}, 20$ on $-80^{\circ}, 24$ on $-75^{\circ}$. Each plate covers an area $10^{\circ} \times 10^{\circ}$. The purpose of this experiment is to examine whether, with this degree of overlapping, it is possible to $(a)$ increase the systematic accuracy of the reference-star system, $(b)$ use fewer stars than have been considered necessary heretofore. He referred to suggestions along these lines made by Dr Stoy(s) at the Evanston Conference.

Dr Dieckvoss stressed the importance of using overlapping plates (cf. the Paris Catalogue of $I I, 755$ stars), and also of measuring plates in strictly orthogonal system in order to strengthen the value of photographic positions.

\title{
REFERENCES
}

(I) F. Schmeidler, Vistas in Astronomy, pt. XI.

(2) G. van Herk, B.A.N. 9, 489, I952.

(3) F. P. Scott, $A . J .60,93$, 1955.

(4) See this volume, p. II 7 .

(5) R. H. Stoy, A.J. 59, 39, I954.

\section{REPORT ON OBSERVATIONS OF SOUTHERN FUNDAMENTAL DECLINATIONS IN PROGRESS AT MT STROMLO OBSERVATORY}

\section{By F. SCHMEIDleR}

The idea of the observations of southern fundamental declinations at Mt Stromlo follows a suggestion first made by Dneprovski (A.N. 24I, 2I9) that the same stars should be observed by the same observer with the same instrument at two stations, one in each hemisphere. Dneprovski's idea was to find out whether the extreme $\Delta \delta_{\delta}$-errors, resulting at that time from most vertical circles and also some meridian circles, were either purely instrumental errors, or real errors in fundamental declinations.

Although it was shown in I933 by Rabe $(A . N . \mathbf{2 4 8}, 369)$ that in most cases the extreme $\Delta \delta_{\delta}$-errors could be removed by applying the right amount of flexure corrections to the respective observations, some unexplained differences remained which were of a smaller amount. According to Kopff $(A . N .269, \mathrm{x} 60)$ the differences between the declinations of $\mathrm{FK}_{3}$ and GC are as large as $0 " 4$ in some parts of the sky, especially at high southern declinations. Therefore it seemed still worth while carrying out Dneprovski's original idea.

The importance of observations of fundamental southern declinations is also supported in view of the revision of $\mathrm{FK}_{3}$, as pointed out recently by Kopff $(M . N$. r r4, 478). There are only a very small number of catalogues of southern fundamental positions, even fewer than there were when the $\mathrm{FK}_{3}$ itself was compiled. Furthermore, Cape and perhaps Cordoba are the only observatories where, at present, regular meridian observations of southern stars are made, so that any systematic errors, by which the observations of one of these two observatories may be affected, enter with very great weight into the final fundamental system.

The author has been observing fundamental declinations with the Munich vertical circle since 1940; his programme of observation of all Auwers stars north of $-30^{\circ}$ declination was completed in I953. The very generous assistance of the Deutsche Forschungsgemeinschaft as well as of the Commonwealth Observatory at Mt Stromlo enabled him to go to Australia in February I954 to make observations of southern fundamental declinations. The actual observations began in May I954 and will probably be finished by September I955.

The proposed programme includes all Auwers stars south of declination $+30^{\circ}$ and also all Zusatzsterne south of the equator. Therefore all Auwers stars in the belt between declinations $-30^{\circ}$ and $+30^{\circ}$ will be observed from both hemispheres and it is hoped to 
get an indication of any systematic differences due to the instrument or to the locality. Unfortunately, not many Zusatzsterne have been observed at Munich, but the writer hopes that the northern Zusatzsterne have been observed by several northern observatories.

Regular observations of the planets Mars, Jupiter, Saturn, Uranus, Neptune, Ceres, Pallas, Juno and Vesta have been made at all available oppositions since I94I and have been continued at Canberra. At a few oppositions the planets Pallas and Juno could not be observed owing to faint magnitude, very southern declination, or both.

The weather conditions at Mt Stromlo have been very favourable as compared with average conditions in Germany. Using even doubtful nights, the author was able to make observations on more than 20 nights each month. The seeing conditions seem to be slightly better than in Europe, although occasionally strong wind causes some trouble.

At the present time (July I955) about $80 \%$ of the intended observations have been made and all these observations have been reduced. The complete confirmation of Rabe's result is already evident, i.e. that after the application of flexure correction as determined by observing stars reflected by mercury, there remain no extreme $\Delta \delta_{\delta}$-errors in the observed declinations. There are systematic and individual differences from the FK 3 positions, but these are of a smaller amount, exceeding $0{ }^{\prime \prime} 5$ only in very few cases.

Detailed results cannot be given at the present time, as the exact value of the latitude can be derived only after a comprehensive discussion of all observations. It is intended to examine the observations for any possible corrections to refraction theory. The observations of planets will be used for deriving the equator point for the Munich observations as well as for those made at Canberra, following the suggestion made by Clemence $(A . J .54$, Io).

The writer will try to derive definite results as soon as possible after completion of the observational work; he hopes to have results ready for publication by the middle of 1956 .

Prof. Witkowski made the following remark:

The original idea of eliminating systematical errors in a fundamental system of declinations by use of observations made in both hemispheres by the same observer and with the same instrument belongs to Prof. B. Zaleski, director of the Poznan Observatory. For this purpose he observed declinations of stars with a transportable Repsold meridian circle and intended to continue his observations in New Zealand. He published his Poznan declination catalogue in 1926 as well as a paper on his method.* His premature death in 1927 prevented him from carrying out the complete programme. The continuation of Zaleski's observations is included in the programme of the Poznan Observatory, but has not been realized till now for lack of financial resources.

\title{
8. PRELIMINARY RESULTS OF ABSOLUTE DETERMINATIONS FROM AZIMUTH OBSERVATIONS AT AN EQUATORIAL STATION
}

\author{
By G. VAN HERK
}

The method proposed by De Sitter and Oort (B.A.N. 8r, r926) consists of a comparison between observed directions of a star when low above the horizon seen from an equatorial station with the computed azimuth. Knowledge of time of observation and geographic position is essential but not critical.

The observations compared with the computed azimuths will yield the zero point of the horizontal circle. The errors in the zero point originating from a systematic error in declination $\left(\Delta \delta_{\delta}\right)$ in clock error and in latitude will be cancelled in the average when sufficient stars in each quadrant of the horizon are observed.

* B. Zaleski, 'Sur les déclinations fondamentales.' Bulletin de l'Académie Polonaise des Sciences et des Lettres, Sér. A, I924. 'Declinations of 486 stars of the Berliner Jahrbuch for the equinox I925.' Id. Sér. A, 1926. 